\title{
A Brief Recap of Tips and Surgical Manoeuvres to Enhance Optimal Outcome of Surgically Placed Peritoneal Dialysis Catheters
}

\author{
Jodie H. Frost and Atul Bagul \\ Transplant Division, III Department, University of Leicester, Leicester LE1 7RH, UK \\ Correspondence should be addressed to Atul Bagul, atulbagul@yahoo.com
}

Received 12 February 2012; Revised 11 June 2012; Accepted 21 June 2012

Academic Editor: Anil K. Agarwal

Copyright $\odot 2012$ J. H. Frost and A. Bagul. This is an open access article distributed under the Creative Commons Attribution License, which permits unrestricted use, distribution, and reproduction in any medium, provided the original work is properly cited.

\begin{abstract}
Background. Peritoneal dialysis (PD) is an effective option of renal replacement therapy for ESRF, offering advantages over haemodialysis. Peritoneal dialysis catheter (PDC) placement is thought to be the key to successful PD and the economic advantages are lost if a patient switches to HD in the 1st year. This paper is a brief document elaborating a recap of published literature, looking at various surgical tips and manoeuvres to enhance optimal outcome of PDC placement. Methods. A search strategy assessing for access team, preoperative antibiotic prophylaxis, type of catheter, catheter exit site, intraoperative catheter trial, optimal time to commence PD, hernia repairs, number of cuffs, catheter-embedding procedures, rectus sheath tunnelling, laparoscopic fixing, omentopexy, omentectomy, the "Y"-Tec system, resection of epiploic appendages, adhesiolysis, a trained surgeon, and perioperative catheter care protocol was used looking at various databases. Findings. The complications of catheterrelated dysfunction can be reduced with advanced planning of access placement, immaculate surgery, and attention to catheter insertion techniques. Conclusion. The success of a peritoneal dialysis programme depends upon functional and durable long term access to the peritoneal cavity; this depends on placement techniques and competent surgeons and psychosocial support to the patient. The various technical tips and manoeuvres elaborated here should be considered options carried out to improve outcome and reduce catheter dysfunction.
\end{abstract}

\section{Introduction}

Peritoneal dialysis $(\mathrm{PD})$ is an effective option of renal replacement therapy for end-stage renal failure, offering advantages such as it being a modality undertaken at home, encourages self-autonomy, and optimises quality of life $[1,2]$. It is well established that access-related morbidity and mortality are significantly lower for PD than for Haemodialysis (HD) [3]. Its other advantages are improved survival benefit during the first 1 to 2 years due to better preservation of residual function and improved blood volume and blood pressure control $[4,5]$. Peritoneal dialysis is less expensive than HD, a cost difference of $\$ 19,000 /$ person/year in the USA (PD828 ) and $\mathfrak{E} 12,000 /$ person/year in the UK (NHSBT-website: http://www.organdonation.nhs.uk/). The success of PD depends upon the presence of a functional and durable long term access to the peritoneal cavity, which depends upon placement technique rather than on catheter design [6].

Thus, catheter placement is thought to be the key of successful PD and the economic advantages are lost if a patient switches to HD in the 1st year [7]. Primary nonfunction rate for PDC in literature is quoted at $10 \%$ [8]. The potential complication for PDC placement includes mechanical flow dysfunction usually associated with malposition, leak, exit-site infection/peritoneal infection, intra-abdominal injuries, and catheter blockages [9]. These complications can be reduced with advanced planning of access placement, immaculate surgery, attention to catheter insertion techniques, and psychosocial support to the patient $[10,11]$. Catheter-related problems like recurrent leaks and hernias, leading to a delay for PD and if not further surgical procedures increase pyscho-social problems in this cohort 
of patients. A multidisciplinary team involving PD nurses, nephrologist, and surgeons will increase the psychosocial support to such a cohort of patients and add to the success of a PD programme.

\section{Objective}

This paper is a brief document elaborating a recap of published literature, looking at various surgical tips and manoeuvres to enhance optimal outcome of peritoneal dialysis catheter placement.

\section{Search Strategy}

Databases: Medline (Pubmed-1966-2011), Cochrane Central Register of Controlled Trials, EMBASE (1974-2011) the Data base of Abstracts of Reviews of Effects (DARE), and Health technology assessment database (HTA).

The search strategy was based on the following:

(i) peritoneal dialysis catheters inserts,

(ii) peritoneal dialysis catheter dysfunction,

(iii) surgical manoeuvres for peritoneal dialysis,

(iv) laparoscopic peritoneal dialysis catheter insertion,

(v) open PDC insertions,

(vi) Tenckhoff PDC catheters,

(vii) peritoneoscopic PDC insertions,

(viii) antibiotic prophylaxis for PDC insertion,

(ix) controversy of PDC,

(x) role of surgeon in PDC placement.

The various tips and surgical manoeuvres to enhance optimal outcome of peritoneal dialysis catheters are discussed as follows.

3.1. Peritoneal Access (PD Access): The Access Team. The Renal Association UK: Peritoneal Access Guidelines: guideline peritoneal dialysis (RA/PAG:PD) [12] and International Society for Peritoneal Dialysis (ISPD) guidelines recommend that each centre should have a dedicated team involved in the implantation and care of peritoneal catheters. (RA/PAG: PD1.1) [13] (ISPD: 1.1) [14].

3.2. Preoperative Antibiotic Prophylaxis. A single dose of preoperative antibiotics is recommended (based on unit microbiology policy)( RA/PAG: PD3.1) [12] (ISPD:3.1) [14] and this reduces the rate of exit-site infection and peritonitis $[9,15-20]$.

3.3. Type of Catheter. RA/PAG: PD5.2 [12] and ISPD: 5.2 suggest that no particular catheter type is proven to be better than another [21]. Though, a curled tip catheter should be used and is more likely to retain position within the pelvis and offers a better drainage compared to a straight tip catheter [22, 23]. Nielsen et al., published a small RCT which demonstrates superiority of the curled Tenckhoff peritoneal dialysis catheter survival as compared to the straight catheter which was due to the higher displacement rate [24]. In addition, straight tip catheters are associated with dialysate inflow discomfort or the "poking pain" which is not reported with curled catheters [22]. An extended catheter system or two-piece extended catheter system can be used to provide remote exit-site locations to the upper abdomen or chest as an indication for obesity, floppy abdominal skin folds, presence of stomas, incontinence, and yeast intertrigo [2528]. Self locating catheters have been described, which show a lower rate of displacements [29-32].

3.4. Catheter Exit-Site. The exit-site should be well planned, ideally on the opposite side of the dominant hand and should not be placed in skin crease, belt line and should be located dependently to the site of cuff placement. (RA/PAG) Many authors have described optimum exit-sites in literature, yet, the ideal situation is that the patient should be marked in a preoperative setting. If this has not been done, then the 3 -step algorithm method could be used to determine exitsite at the time of the operation [10]. It is to design a laterally directed exit-site that places the superficial cuff $4 \mathrm{~cm}$ from the exit wound and also eliminates concerns for cuff extrusion from tubing resiliency forces. In brief, the three steps include the following Step 1: scribe arc from vertical to lateral plane using catheter as compass from point $2 \mathrm{~cm}$ external of superficial cuff. Step 2: mark exit-site at junction of medial 2/3rd and lateral 1/3rd of arc. Step 3: indicate tunnel tract shape by bending catheter over from point $4 \mathrm{~cm}$ external of superficial cuff to exit-site.

3.5. Intraoperative Catheter Trial. Various volumes have been used but we prefer to use half a bag of PD fluid to check for instillation and drainage. This is a very important and crucial step as this manoeuvre does predict successful function (inflow and outflow) of the PDC postoperatively. In addition, further small volume exchanges can be carried out in patients who have undergone extensive adhesiolysis until draining dialysate is no longer blood stained [9]. In addition, it should also be used to test the tightness of catheter at the peritoneum, thus excluding a possible dialysate leak.

3.6. Optimal Time to Commence PD. An approximate 2-week delay (RA/PAG: PD2.1) [12] (ISPD: 2.1) [14] results in the less likelihood of exit-site infection and dialysate leak $[9,33]$, though this is based on evidence from mostly retrospective studies. Reported leak rates are significantly lower for all documented laparoscopic series, even when catheters are used straight away $[1,34-38]$. Purse string sutures at the cuff to anchor have been described during open surgery and do reduce the risk of early leaks $[39,40]$. Yang et al., did not show higher risk of catheter-related problems in PD patients starting dialysis less than 2 weeks (earlier group $(2.0 \pm 2.7$ days) versus late group $(40.6 \pm 42.8$ days): $14.6 \%$ and $13.1 \%$, resp. developed catheter-related complications within 6 months) [41]. Currently, a randomised control 
study (timely PD study) has been set up to address the influence of different break in periods [42].

3.7. Hernia Repairs. Utmost importance should be given to the consent, where the patient should be counselled for hernia repair found at the time of the operation. GracíaUreña et al. in their article yet stress the importance of identifying and repairing of hernias prior to commencing PD [43]. The hernioplasty should be performed using prosthetic material as a tension-free repair, hence, to reduce the risk of recurrence in peritoneal dialysis patient [43-45]. This may lead to a small draw back being a delay in initial catheter use for peritoneal dialysis and patients should be warned about interim haemodialysis $[9,10]$, though literature supports successful immediate restart of PD [45].

3.8. Number of Cuffs. Controversy lies with either using a catheter with a single or two cuffs. A single-cuffed catheter logically avoids a second foreign body being placed in the less-vascularised subcutaneous tissue, thereby reducing the risk of cuff infections or colonisation near the exit-site [9]. Eklund et al., published in a small RCT (30 per arm) that there to be no significant difference between catheters with single or double cuffs with respect to catheter survival, episodes of peritonitis, and exit-site infections [46]. Though, some published lines of literature prefer two-cuffed catheters as they reduce risk of peritonitis in the long run [9]. Nessim et al., reported double cuff were associated with reduced peritonitis rates between 1996 and 2000, though after 2000 this risk disappeared and was probably due to influence of prophylactic measures or increase in experience on the risk of infection over time [47]. The Cochrane Review did not find any advantage for straight versus coiled catheters, single or double cuff, and median or lateral incision [21] and the ISPD guidelines recommend no difference (ISPD: 5.2) [14].

3.9. Catheter-Embedding Procedures. These procedures were initially described by Moncrief et al., [48], where the procedure entails the external limb of the dialysis catheter is embedded subcutaneously at the time of implantation. After about 3-5 weeks interval, exteriorization is carried out under local anaesthesia in the day case setting. Brown et al., recommend insertion 6 weeks to 5 months ahead of the need for PD to maximize catheter survival. The authors looked at three groups of patients starting PD 11-47 days, 48-133 days, and more than 133 days after placement of embedded catheters. The time to catheter loss was shortest in group 1 and longest in group 2 and suggested that mechanical complications and catheter loss are associated with the length of time a catheter is embedded [49]. The concept speculated reduced catheter-related infection due to firm tissue ingrowth of the cuff and the absence of a biofilm. With time and use of antibiotic prophylaxis these procedures had initially lost their popularity. But in reality it may be a useful strategy to lock a patient into peritoneal so as to avoid crash haemodialysis with a tunnelled catheter if sudden decline in renal function were to occur. The technique of embedding allows efficient surgical scheduling of catheter placement as a planned procedure which can lead to a significant longer catheter life [50] and fewer earlier complications [51].

3.10. Rectus Sheath Tunnelling. This leads to creation of a long musculofascial tunnel in a craniocaudal direction, thus maintaining pelvic orientation of the catheter tip [5255]. This manoeuvre leads to reduced risk of pericannular leaks and hernias [10]. A detailed step-by-step technique for rectus sheath tunnelling has been described by Crabtree [10] To facilitate a further reduction of leaks during transrectus placement, a fixation suture around the catheter at the anterior rectus sheath may be placed, with the cuff being secured at this fascial layer [56-58]. Attaluri et al., data clearly showed a significant improvement in PD catheter function using omentopexy and rectus sheath tunnelling [58].

3.11. Laparoscopic Fixation. Laparoscopic fixation to prevent catheter tip migration may be carried out where the catheter tip is fixed to the lower abdomen or pelvis $[59,60]$. The drawback here is an increased risk of obstructing adhesions, potential for internal hernias and suture knot, a nidus for persistent infection; proper rectus sheath tunnelling and placement of the deep cuff are the key to reducing catheter tip migration [10].

3.12. Omentopexy. Omentopexy is usually carried out when partial or complete catheter obstruction/displacement is produced due to omental entrapment. The procedure involves a laparoscopic omental tack up, which is performed high in the abdomen at the Palmer point [61] or to the falciform ligament [62]. This procedure is not limited to rescuing catheters from omental entrapment and does play a valuable role in selective prophylactic omentopexy as an adjunctive procedure to catheter implantation as assessed by an intraoperative catheter trial [6] and especially when the omentum extends to the pelvis in a juxtaposition of the peritoneal catheter tip [63].

3.13. Omentectomy. Omentectomy is usually avoided as an omentopexy is a much quicker and cost-effective procedure with an equivalent outcome $[10,64]$. Persistent dysfunction problems due to omental wraps and failed omentopexy warrant an omentectomy though rare.

3.14. The Modified "Y"-Tec System. This system permits the procedure to be carried out with either a single $5 \mathrm{~mm}$ lapport or a $2.2 \mathrm{~mm}$ peritoneoscope, thus leading to reduced incisions, leaks, and hernias. In addition, in particular with the laparoscope, an adequate and thorough diagnostic look can be carried out in particular for redo/exchange of PD catheters. Additional ports can be inserted to carry out procedure like adhesiolysis, omentopexy, and peritoneal biopsies [65].

3.15. Resection of Epiploic Appendages. Epiploic appendages are an infrequent cause of catheter dysfunction and can be identified during the intraoperative catheter trial irrigation 
test. Once identified the offending epiploic (seen blocking the catheter during an outflow check) appendages indicate a laparoscopic resection [66].

3.16. Adhesiolysis. Adhesions play a significant role in catheter displacement, thus dysfunction. Extensive dissection is neither necessary nor desirable to mobilise and divide every single adhesion. Abdominal wall adhesion above the level of the pelvis will not interfere and is best left alone. Laparoscopic dissection can be carried out using either scissor with diathermy, hook diathermy or ultrasonic shears. Limiting adhesiolysis to that necessary avoids visceral injury, haemorrhage, and reformation of adhesions which will eventually compromise the peritoneal cavity [10].

3.17. A Trained Surgeon. Surgical outcomes strongly corelate with training during residency [67]. RA/PAG PD6.1 [12] recommend that $\mathrm{PD}$ catheter insertion training should be available to all trainees with an interest [68]. Placement of a PDC by an experienced surgeon is strongly recommended to reduce complications [69]. Repetition of the procedure is needed for surgical proficiency $[67,70]$ and the number quoted is 20-40 procedures before an acceptable level of skill can be obtained [70, 71]. RA/PAG PD6.2 [12] recommend that $\mathrm{PD}$ catheter insertion should not be delegated to inexperienced unsupervised operators [13].

3.18. Patient Selection. Surgical placement of PDC should be the standard for complicated patients such as PDC revisions and patients with hernias and redo abdominal surgery. The procedure should be carried out as a laparoscopic placement (direct visualisation; ISPD guideline 4.1) under a general anaesthesia as problems such as adhesions and hernias can be dealt with at the time of surgery $[10,15,28,61,65]$. The anaesthetic requirement depends on the technique selected, which is influenced by the patients characteristics (ISPD: 5.1 ) [14] and thus for complicated patients general anaesthesia is preferred.

ISPD guideline 4.1 recommend that local expertise at individual centres should govern the choice of method of PDC placement, with timely surgical support available for the review of PD patients (ISPD: 4.4) [14]. There is no RCT evidence to support one method of insertion over another; however, the method needs be determined by patient characteristics (ISPD: 4.4) [14]. Indeed, some data show that laparoscopic PDC implantation may be superior to other methods in patients with pervious operation $[58,72]$, though results of the RCT LOCI trial are awaited [73].

3.19. Perioperative Catheter Care Protocol. RA/PAG PD 3.1 and ISPD 3.1 recommend that renal units should have clear protocols for perioperative catheter care addressing the following $[12,15]$ :

(a) Preoperatively_ check for hernias, screen for MRSA, and nasal carriage of Staphylococcus aureus, identifying a catheter of a suitable length, and mark the appropriate exit-site sitting and standing. (b) Before-implantation-bowel preparation with laxatives, ensuring bladder emptying, administration of prophylactic antibiotics, and surgical site preparation according to NICE guidance [18].

(c) Aftert-procedure-catheter flush and cap off using suitable dialysate, exit-site covered with a suitable nonocclusive dressing and if possible not disturbed for 5-10 days, immobilisation of the catheter, patient discharged home with supply of aperients with advice as how to recognise potential complications. Intraperitoneal administration of heparin catheter flushes prevents fibrin formation in CAPD patients and may have added benefits [74].

Once the catheter is placed and until healing is completed, the dressing changes should be addressed by a dialysis followup nurse using sterile technique.

3.20. Contraindications for PDC (http://www.kidney.org/professionals/kdoqi/guidelines/). Absolute contraindications for PD include (guideline 30: NKF KDOQI guidelines for peritoneal dialysis adequacy)

(a) documented loss of peritoneal function or extensive abdominal adhesions that limit dialysate flow,

(b) uncorrectable mechanical defects that prevent effective PD or increase the risk of infection (e.g., surgically irreparable hernia, omphalocele, gastroschisis, diaphragmatic hernia, and bladder exstrophy).

Relative contraindications for PD include the following. (Guideline 31: NKF KDOQI).

(a) Fresh intra-abdominal foreign bodies (e.g., 4-month wait after abdominal vascular prostheses, recent ventricular-peritoneal shunt).

(b) Peritoneal leaks.

(c) Body size limitations.

(d) Intolerance to PD volumes necessary to achieve adequate $\mathrm{PD}$ dose.

(e) Inflammatory or ischemic bowel disease.

(f) Abdominal wall or skin infection.

(g) Morbid obesity (in short individuals).

(h) Severe malnutrition.

(i) Frequent episodes of diverticulitis.

\section{Conclusions}

The success of a peritoneal dialysis programme depends upon functional and durable long term access to the peritoneal cavity; this depends on placement techniques and competent surgeons. The various technical tips and manoeuvres elaborated here should be considered options carried out to improve outcome and reduce catheter dysfunction. 


\section{References}

[1] J. H. Crabtree and R. J. Burchette, "Effective use of laparoscopy for long-term peritoneal dialysis access," American Journal of Surgery, vol. 198, no. 1, pp. 135-141, 2009.

[2] S. S. A. Fenton, D. E. Schaubel, M. Desmeules et al., "Hemodialysis versus peritoneal dialysis: a comparison of adjusted mortality rates," American Journal of Kidney Diseases, vol. 30, no. 3, pp. 334-342, 1997.

[3] S. Mujais and C. Holmes, "Modern peritoneal dialysis: concepts and approaches," Kidney International, vol. 70, supplement 103, pp. S1-S2, 2006.

[4] R. Gokal, P. G. Blake, J. Passlick-Deetjen, T. P. Schaub, S. Prichard, and J. M. Burkart, "What is the evidence that peritoneal dialysis is underutilized as an ESRD therapy?" Seminars in Dialysis, vol. 15, no. 3, pp. 149-161, 2002.

[5] E. F. Vonesh, J. J. Snyder, R. N. Foley, and A. J. Collins, "Mortality studies comparing peritoneal dialysis and hemodialysis: what do they tell us?" Kidney International, vol. 70, supplement 103, pp. S3-S11, 2006.

[6] S. C. Jwo, K. S. Chen, C. C. Lee, and H. Y. Chen, "Prospective randomized study for comparison of open surgery with laparoscopic-assisted placement of Tenckhoff peritoneal dialysis catheter-a single centre experience and literature review," Journal of Surgical Research, vol. 159, pp. 489-496, 2010.

[7] Y. C. Shih, A. Guo, P. M. Just, and S. Mujais, "Impact of initial dialysis modality and modality switches on Medicare expenditures of end-stage renal disease patients," Kidney International, vol. 68, pp. 319-329, 2005.

[8] A. Danielsson, "The controversy of placement of peritoneal dialysis catheters," Peritoneal Dialysis International, vol. 27, no. 2, pp. 153-154, 2007.

[9] A. H. Gajjar, D. H. Rhoden, P. Kathuria, R. Kaul, A. D. Udupa, and W. C. Jennings, "Peritoneal dialysis catheters: laparoscopic versus traditional placement techniques and outcomes," American Journal of Surgery, vol. 194, no. 6, pp. 872-876, 2007.

[10] J. H. Crabtree, "Selected best demonstrated practices in peritoneal dialysis access," Kidney International, vol. 70, no. 103, pp. S27-S37, 2006.

[11] A. Guo and S. Mujais, "Patient and technique survival on peritoneal dialysis in the United States: evaluation in large incident cohorts," Kidney International, vol. 64, supplement 88, pp. S3-S12, 2003.

[12] M. Wilkie, S. Jenking, and B. Shrestha, The Renal Association Clinical Guideline UK Peritoneal Access Guidelines, 5th edition, 2009, http //www.renal.org/Clinical/GuidelinesSection/PeritonealDialysis.aspx.

[13] M. J. Flanigan and R. Gokal, "Peritoneal catheters and exitsite practices toward optimum peritoneal access: a review of current developments," Peritoneal Dialysis International, vol. 25, no. 2, pp. 132-139, 2005.

[14] A. Figueiredo, B. L. Goh, S. Jenkins et al., "Clinical practice guidelines for peritoneal access," Peritoneal Dialysis International, vol. 30, no. 4, pp. 424-429, 2010.

[15] L. Salman and A. Asif, "Antibiotic prophylaxis: is it needed for dialysis access procedures?" Seminars in Dialysis, vol. 22, no. 3, pp. 297-299, 2009.

[16] D. Leaper, S. Burman-Roy, A. Palanca et al., "Prevention and treatment of surgical site infection: summary of NICE guidance," BMJ, vol. 337, article a1924, 2008.
[17] M. F. Gadallah, G. Ramdeen, J. Mignone, D. Patel, L. Mitchell, and S. Tatro, "Role of preoperative antibiotic prophylaxis in preventing postoperative peritonitis in newly placed peritoneal dialysis catheters," American Journal of Kidney Diseases, vol. 36, no. 5, pp. 1014-1019, 2000.

[18] G. F. M. Strippoli, A. Tong, D. Johnson, F. P. Schena, and J. C. Craig, "Antimicrobial agents to prevent peritonitis in peritoneal dialysis: a systematic review of randomized controlled trials," American Journal of Kidney Diseases, vol. 44, no. 4, pp. 591-603, 2004.

[19] B. Piraino, G. R. Bailie, J. Bernardini et al., "Peritoneal dialysisrelated infections recommendations: 2005 update," Peritoneal Dialysis International, vol. 25, no. 2, pp. 107-131, 2005.

[20] B. Piraino, J. Bernardini, E. Brown et al., "ISPD position statement on reducing the risks of peritoneal dialysis-related infections," Peritoneal Dialysis International, vol. 31, no. 6, pp. 614-630, 2011.

[21] G. F. Strippoli, A. Tong, D. Johnson, F. P. Schena, and J. C. Craig, "Catheter type, placement and insertion techniques for preventing peritonitis in peritoneal dialysis patients," Cochrane Database of Systematic Reviews, no. 4, article CD004680, 2004.

[22] W. C. Lye, N. W. Kour, J. C. Van Der Straaten, S. O. Leong, and E. J. C. Lee, "A prospective randomized comparison of the Swan neck, coiled, and straight Tenckhoff catheters in patients on CAPD," Peritoneal Dialysis International, vol. 16, no. 1, pp. S333-S335, 1996.

[23] A. M. Akyol, C. Porteous, and M. W. Brown, "A comparison of two types of catheters for continuous ambulatory peritoneal dialysis (CAPD)," Peritoneal Dialysis International, vol. 10, no. 1, pp. 63-66, 1990.

[24] P. K. Nielsen, C. Hemmingsen, S. U. Friis, J. Ladefoged, and K. Olgaard, "Comparison of straight and curled Tenckhoff peritoneal dialysis catheters implanted by percutaneous technique: a prospective randomized study," Peritoneal Dialysis International, vol. 15, no. 1, pp. 18-21, 1995.

[25] J. H. Crabtree, "Is the Tenckhoff catheter still the first choice for use with peritoneal dialysis?" Seminars in Dialysis, vol. 24, no. 4, pp. 447-448, 2011.

[26] J. H. Crabtree, "Extended peritoneal dialysis catheters for upper abdominal wall exit sites," Peritoneal Dialysis International, vol. 24, no. 3, pp. 292-294, 2004.

[27] Z. J. Twardowski, B. F. Prowant, W. K. Nichols, K. D. Nolph, and R. Khanna, "Six-year experience with swan neck presternal peritoneal dialysis catheter," Peritoneal Dialysis International, vol. 18, no. 6, pp. 598-602, 1998.

[28] J. H. Crabtree and A. Fishman, "Laparoscopic implantation of swan neck presternal peritoneal dialysis catheters," Journal of Laparoendoscopic and Advanced Surgical Techniques A, vol. 13, no. 2, pp. 131-137, 2003.

[29] N. Di Paolo, G. Petrini, G. Garosi, U. Buoncristiani, S. Brardi, and G. Monaci, "A new self-locating peritoneal catheter," Peritoneal Dialysis International, vol. 16, no. 6, pp. 623-627, 1996.

[30] N. Di Paolo, E. Sansoni, F. Cappelletti et al., "The self-locating catheter: review and cost analysis," International Journal of Artificial Organs, vol. 29, no. 1, pp. 113-122, 2006.

[31] N. Di Paolo, L. Capotondo, E. Sansoni et al., "The self-locating catheter: clinical experience and follow-up," Peritoneal Dialysis International, vol. 24, no. 4, pp. 359-364, 2004.

[32] Z. J. Twardowski, "History of peritoneal access development," International Journal of Artificial Organs, vol. 29, no. 1, pp. 240, 2006. 
[33] N. Dombros, M. Dratwa, M. Feriani et al., "European best practice guidelines for peritoneal dialysis. 3 Peritoneal access," Nephrology Dialysis Transplantation, vol. 20, pp. ix8-ix12, 2005.

[34] S. C. Schmidt, C. Pohle, J. M. Langrehr, G. Schumacher, D. Jacob, and P. Neuhaus, "Laparoscopic-assisted placement of peritoneal dialysis catheters: implantation technique and results," Journal of Laparoendoscopic and Advanced Surgical Techniques, vol. 17, no. 5, pp. 596-599, 2007.

[35] J. Y. Wang, F. M. Chen, T. J. Huang et al., "Laparoscopic assisted placement of peritoneal dialysis catheters for selected patients with previous abdominal operation," Journal of Investigative Surgery, vol. 18, no. 2, pp. 59-62, 2005.

[36] P. Soontrapornchai and T. Simapatanapong, "Comparison of open and laparoscopic secure placement of peritoneal dialysis catheters," Surgical Endoscopy and Other Interventional Techniques, vol. 19, no. 1, pp. 137-139, 2005.

[37] R. Maio, N. Figueiredo, and P. Costa, "Laparoscopic placement of Tenckhoff catheters for peritoneal dialysis: a safe, effective, and reproducible procedure," Peritoneal Dialysis International, vol. 28, no. 2, pp. 170-173, 2008.

[38] A. Keshvari, I. Najafi, M. Jafari-Javid, M. Yunesian, R. Chaman, and M. N. Taromlou, "Laparoscopic peritoneal dialysis catheter implantation using a Tenckhoff trocar under local anesthesia with nitrous oxide gas insufflation," American Journal of Surgery, vol. 197, no. 1, pp. 8-13, 2009.

[39] Y. I. Jo, S. K. Shin, J. H. Lee, J. O. Song, and J. H. Park, "Immediate initiation of CAPD following percutaneous catheter placement without break-in procedure," Peritoneal Dialysis International, vol. 27, no. 2, pp. 179-183, 2007.

[40] B. G. Stegmayr, "Three purse-string sutures allow immediate start of peritoneal dialysis with a low incidence of leakage," Seminars in Dialysis, vol. 16, no. 4, pp. 346-348, 2003.

[41] Y. F. Yang, H. J. Wang, C. C. Yeh, H. H. Lin, and C. C. Huang, "Early initiation of continuous ambulatory peritoneal dialysis in patients undergoing surgical implantation of Tenckhoff catheters," Peritoneal Dialysis International, vol. 31, no. 5, pp. 551-557, 2011.

[42] D. Ranganathan, R. Baer, R. G. Fassett et al., "Randomised Controlled Trial to determine the appropriate time to initiate peritoneal dialysis after insertion of catheter to minimise complications (Timely PD study)," BMC Nephrology, vol. 11, no. 1 , article $11,2010$.

[43] M. A. García-Ureña, C. R. Rodríguez, V. V. Ruiz et al., "Prevalence and management of hernias in peritoneal dialysis patients," Peritoneal Dialysis International, vol. 26, no. 2, pp. 198-202, 2006.

[44] E. Gianetta, D. Civalleri, A. Serventi et al., "Anterior tensionfree repair under local anesthesia of abdominal wall hernias in continuous ambulatory peritoneal dialysis patients," Hernia, vol. 8, no. 4, pp. 354-357, 2004.

[45] J. H. Crabtree, "Hernia repair without delay in initiating or continuing peritoneal dialysis," Peritoneal Dialysis International, vol. 26, no. 2, pp. 178-182, 2006.

[46] B. Eklund, E. Honkanen, L. Kyllönen, K. Salmela, and A. R. Kala, "Peritoneal dialysis access: Prospective randomized comparison of single-cuff and double-cuff straight Tenckhoff catheters," Nephrology Dialysis Transplantation, vol. 12, no. 12, pp. 2664-2666, 1997.

[47] S. J. Nessim, J. M. Bargman, and S. V. Jassal, "Relationship between double-cuff versus single-cuff peritoneal dialysis catheters and risk of peritonitis," Nephrology Dialysis Transplantation, vol. 25, no. 7, pp. 2310-2314, 2010.
[48] J. W. Moncrief, R. P. Popovich, L. J. Broadrick, Z. Z. He, E. E. Simmons, and R. A. Tate, "The Moncrief-Popovich catheter: a new peritoneal access technique for patients on peritoneal dialysis," ASAIO Journal, vol. 39, no. 1, pp. 62-65, 1993.

[49] P. A. Brown, B. B. McCormick, G. Knoll et al., "Complications and catheter survival with prolonged embedding of peritoneal dialysis catheters," Nephrology Dialysis Transplantation, vol. 23, no. 7, pp. 2299-2303, 2008.

[50] F. C. Prischl, M. Wallner, H. Kalchmair, F. Povacz, and R. Kramar, "Initial subcutaneous embedding of the peritoneal dialysis catheter-a critical appraisal of this new implantation technique," Nephrology Dialysis Transplantation, vol. 12, no. 8, pp. 1661-1667, 1997.

[51] S. R. Ash, "Chronic peritoneal dialysis catheters: overview of design, placement, and removal procedures," Seminars in Dialysis, vol. 16, no. 4, pp. 323-334, 2003.

[52] G. Ogunc, "Minilaparoscopic extraperitoneal tunneling with omentopexy: a new technique for CAPD catheter placement," Peritoneal Dialysis International, vol. 25, no. 6, pp. 551-555, 2005.

[53] B. Draganic, A. James, M. Booth, and J. S. Gani, "Comparative experience of a simple technique for laparoscopic chronic ambulatory peritoneal dialysis catheter placement," Australian and New Zealand Journal of Surgery, vol. 68, no. 10, pp. 735739, 1998.

[54] G. H. Poole and P. Tervit, "Laparoscopic Tenckhoff catheter insertion: a prospective study of a new technique," Australian and New Zealand Journal of Surgery, vol. 70, no. 5, pp. 371373, 2000.

[55] M. Comert, A. Borazan, E. Kulah, and B. H. Uçan, "A new laparoscopic technique for the placement of a permanent peritoneal dialysis catheter: the preperitoneal tunneling method," Surgical Endoscopy and Other Interventional Techniques, vol. 19, no. 2, pp. 245-248, 2005.

[56] B. Eklund, P. H. Groop, L. Halme, E. Honkanen, and A. R. Kala, "Peritoneal dialysis access: a comparison of peritoneoscopic and surgical insertion techniques," Scandinavian Journal of Urology and Nephrology, vol. 32, no. 6, pp. 405-408, 1998.

[57] D. G. Oreopoulos, G. Baird-Helfrich, R. Khanna et al., "Peritoneal catheters and exit-site practices: current recommendations," Peritoneal Dialysis Bulletin, vol. 7, no. 3, pp. 130138, 1987.

[58] V. Attaluri, C. Lebeis, S. Brethauer, and S. Rosenblatt, "Advanced laparoscopic techniques significantly improve function of peritoneal dialysis catheters," Journal of the American College of Surgeons, vol. 211, no. 6, pp. 699-704, 2010.

[59] D. I. Watson, D. Paterson, and K. Bannister, "Secure placement of peritoneal dialysis catheters using a laparoscopic technique," Surgical Laparoscopy, Endoscopy and Percutaneous Techniques, vol. 6, no. 1, pp. 35-37, 1996.

[60] D. L. Heithold, T. D. Duncan, J. G. White, and G. W. Lucas, "Laparoscopic placement of peritoneal dialysis catheters with medial umbilical fold tunnel formation," Surgical Rounds, vol. 20, pp. 310-314, 1997.

[61] J. H. Crabtree and A. Fishman, "Selective performance of prophylactic omentopexy during laparoscopic implantation of peritoneal dialysis catheters," Surgical Laparoscopy, Endoscopy and Percutaneous Techniques, vol. 13, no. 3, pp. 180-184, 2003.

[62] J. H. Crabtree and A. Fishman, "Laparoscopic epiplopexy of the greater omentum and epiploic appendices in the salvaging of dysfunctional peritoneal dialysis catheters," Surgical 
Laparoscopy, Endoscopy and Percutaneous Techniques, vol. 6, no. 3, pp. 176-180, 1996.

[63] J. H. Crabtree and A. Fishman, "A laparoscopic method for optimal peritoneal dialysis access," American Surgeon, vol. 71, no. 2, pp. 135-143, 2005.

[64] J. H. Crabtree and A. Fishman, "Laparoscopic omentectomy for peritoneal dialysis catheter flow obstruction: a case report and review of the literature," Surgical Laparoscopy and Endoscopy, vol. 9, no. 3, pp. 228-233, 1999.

[65] A. Bagul, L. C. Firmin, U. M. Thiyagarajan, and M. L. Nicholson, "Laparoscopic reinsertion/exchanges of peritoneal dialysis catheters using the modified y-TECß system," Annals of the Royal College of Surgeons of England, vol. 93, no. 4, pp. 322-323, 2011.

[66] J. H. Crabtree, "Rescue and salvage procedures for mechanical and infectious complications of peritoneal dialysis," International Journal of Artificial Organs, vol. 29, no. 1, pp. 67-84, 2006.

[67] R. H. Bell, T. W. Biester, A. Tabuenca et al., "Operative experience of residents in US general surgery programs: a gap between expectation and experience," Annals of Surgery, vol. 249, no. 5, pp. 719-724, 2009.

[68] J. S. Berns and W. C. O'Neill, "Performance of procedures by nephrologists and nephrology fellows at U.S. nephrology training programs," Clinical Journal of the American Society of Nephrology, vol. 3, no. 4, pp. 941-947, 2008.

[69] L. P. Wong, S. E. Liebman, K. A. Wakefield, and S. Messing, "Training of surgeons in peritoneal dialysis catheter placement in the United States: a national survey," Clinical Journal of the American Society of Nephrology, vol. 5, no. 8, pp. 1439-1446, 2010.

[70] A. M. Van Rij, J. R. Mcdonald, R. A. Pettigrew, M. J. Putterill, C. K. Reddy, and J. J. Wright, "CUSUM as an aid to early assessment of the surgical trainee," British Journal of Surgery, vol. 82, no. 11, pp. 1500-1503, 1995.

[71] J. H. Crabtree, "Who should place peritoneal dialysis catheters?" Peritoneal Dialysis International, vol. 30, no. 2, pp. 142-150, 2010.

[72] J. H. Crabtree and R. J. Burchette, "Effect of prior abdominal surgery, peritonitis, and adhesions on catheter function and long-term outcome on peritoneal dialysis," American Surgeon, vol. 75, no. 2, pp. 140-147, 2009.

[73] S. M. Hagen, A. M. van Alphen, J. N. M. IJzermans, and F. J. Dor, "Laparoscopic versus open peritoneal dialysis catheter insertion, the LOCI-trial: a study protocol," BMC Surgery, vol. 11 , article 35, 2011.

[74] T. Tabata, H. Shimada, M. Emoto et al., "Inhibitory effect of heparin and/or antithrombin III on intraperitoneal fibrin formation in continuous ambulatory peritoneal dialysis," Nephron, vol. 56, no. 4, pp. 391-395, 1990. 


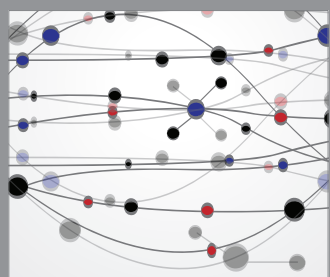

The Scientific World Journal
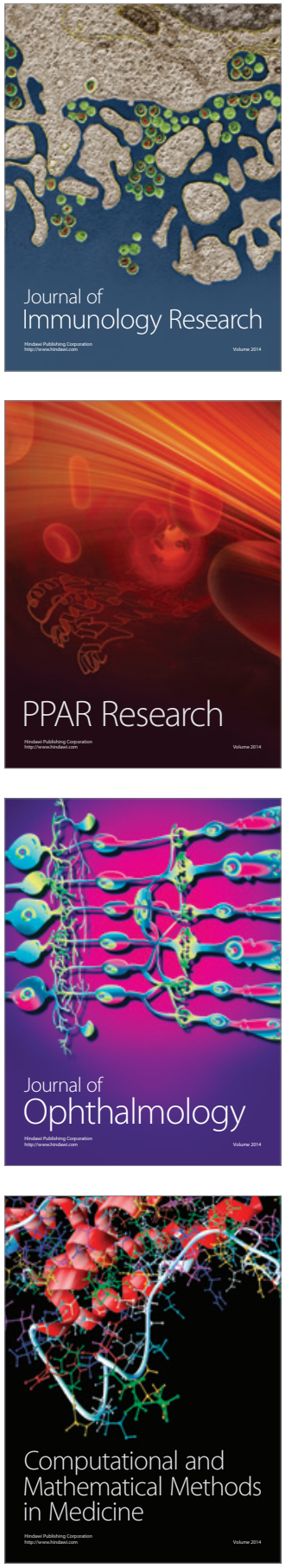

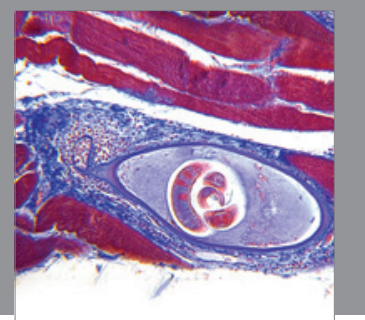

Gastroenterology

Research and Practice
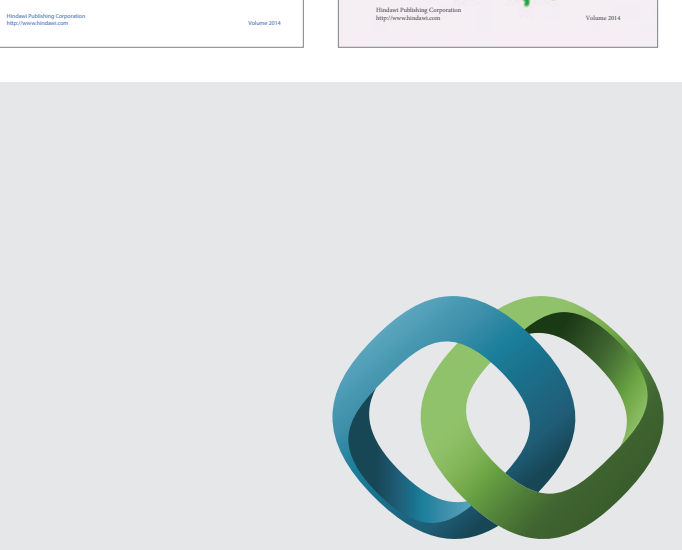

\section{Hindawi}

Submit your manuscripts at

http://www.hindawi.com
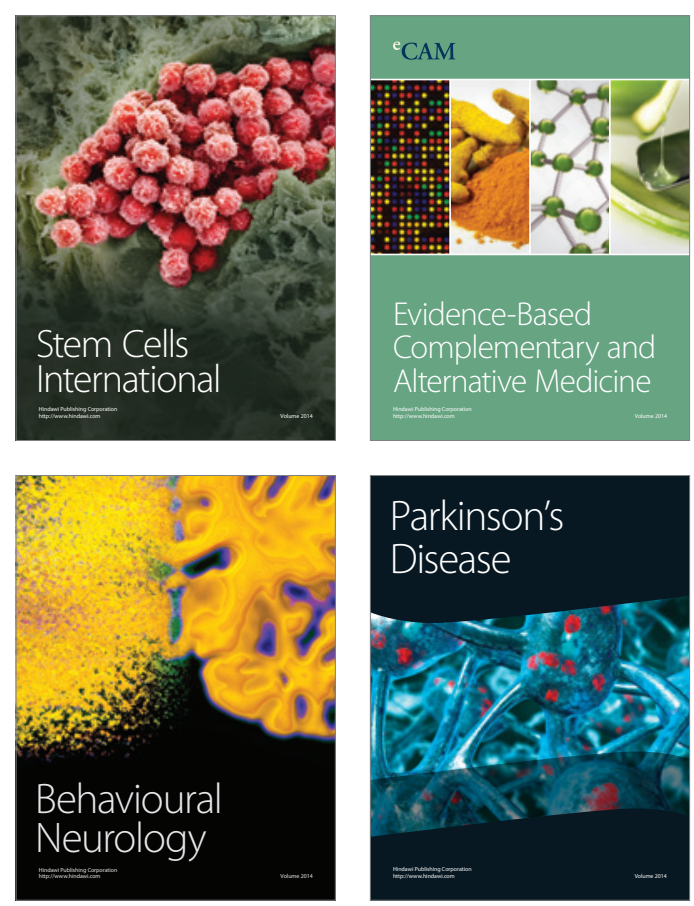

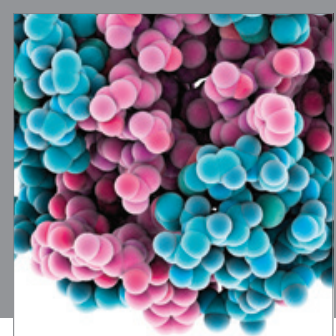

Journal of
Diabetes Research

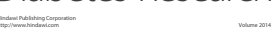

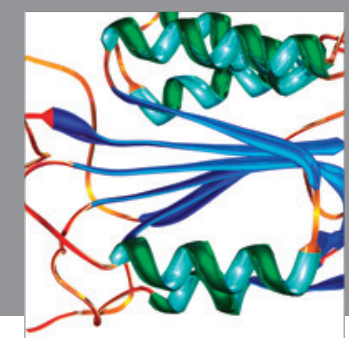

Disease Markers
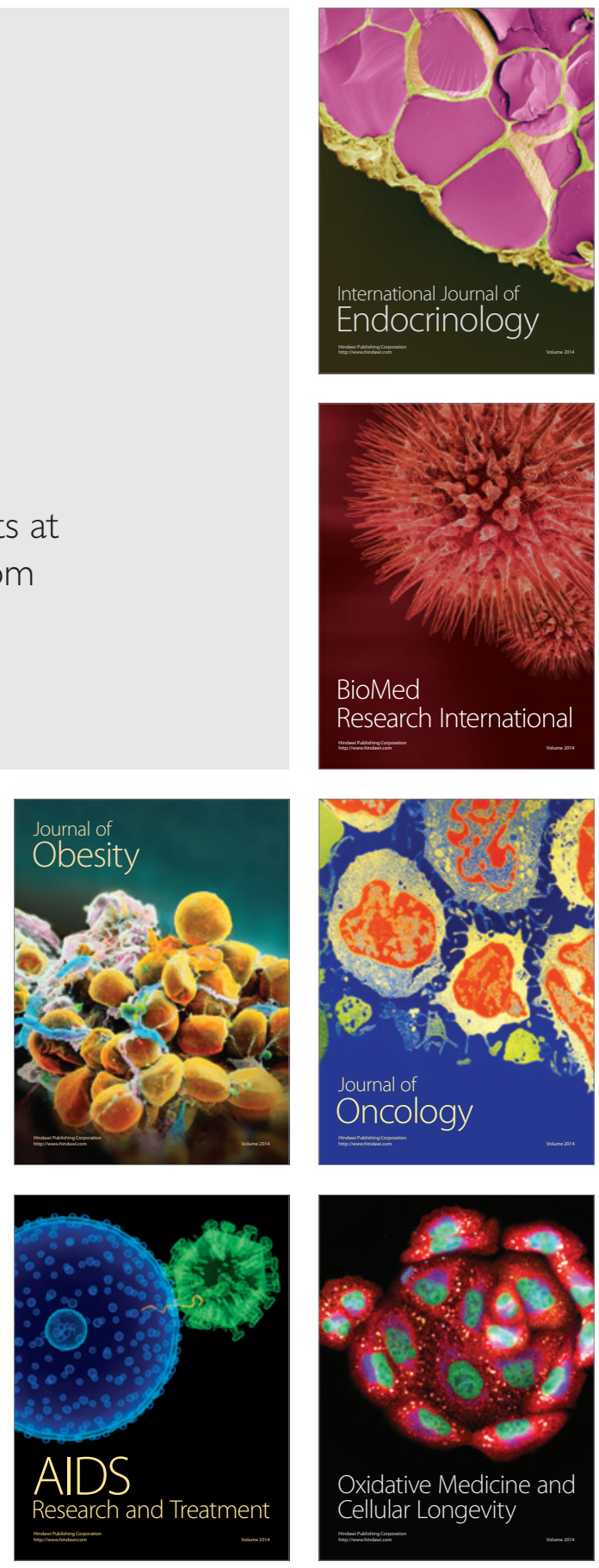